\title{
Pacific
}

Journal of

Mathematics

APPLICATIONS OF SUBORDINATION CHAINS TO STARLIKE MAPPINGS IN $\mathbb{C}^{n}$

MARTIN CHUAQUI

Volume $168 \quad$ No. 1

March 1995 


\section{APPLICATIONS OF SUBORDINATION CHAINS TO STARLIKE MAPPINGS IN $\mathbb{C}^{n}$}

\section{Martin Chuaqui}

We use the work of Pfaltzgraff on subordination chains in $\mathbb{C}^{n}$ to recover a growth theorem for starlike mappings of the unit ball established recently by Barnard, FitzGerald and Gong. We also introduce a class of strongly starlike maps for which we construct, aided by the aforementioned technique, an explicit quasiconformal extension to $\mathbb{C}^{n}$. Several examples are discussed at the end.

1. Introduction. Let $f$ be a univalent map of the unit disc, with $f(0)=0$ and $f^{\prime}(0)=1$. The celebrated Koebe theorem asserts that the image of $f$ contains a disc about the origin of radius $1 / 4,1 / 4$ being sharp. This theorem has no analogue in several complex variables, whether one deals with normalized univalent maps of the unit ball $B^{n}$ or the polydisc. By normalized we mean fixing the origin and having the identity as differential at that point. In particular, the classical growth theorem in dimension 1

$$
\frac{|z|}{(1+|z|)^{2}} \leq|f(z)| \leq \frac{|z|}{(1-|z|)^{2}}
$$

is no longer valid in higher dimensions. Remarkably, (1.1) persists for arbitrary $n$ when considering the subclass of starlike maps of $B^{n}$, as Barnard, FitzGerald and Gong have recently shown [BFG 1]. The result is sharp. Recall that a map is called starlike if the image is starlike with respect to the origin. Suffridge has given the following alternative local characterization: let $w(z)=(D f)^{-1}(f)$, where the differential and the function are evaluated at $z$. Then $f$ is starlike if and only if

$$
\operatorname{Re}\langle\bar{z}, w(z)\rangle \geq 0 \text {. }
$$

Here $\langle a, b\rangle=\sum a_{i} b_{i}$ for $a, b \in \mathbb{C}^{n}[\mathbf{S} \mathbf{1}]$. When $n=1$ then (1.2) recovers the condition $\operatorname{Re}\left\{z \frac{f^{\prime}}{f}\right\} \geq 0$. The proof in [BFG 1] uses (1.2) 
to estimate $\frac{d}{d s}|f|^{2}$ along the (arclength parametrized) preimages in the ball of straight line segments in the image $f\left(B^{n}\right)$.

On the other hand, the bounds in (1.1) hold for a more general class of functions, namely those associated to subordination chains. Let $f(z, t)$ be a family of functions on $B^{n} \times[0, \infty)$, holomorphic in $z$ for each $t$, normalized so that $f(0, t)=0$ and $D f(0, t)=e^{t} \mathrm{I}$. We say that $f(z, t)$ forms a subordination chain provided that for each $s, t$, $0 \leq s \leq t$, there exists a (Schwarz) function $v(z, s, t)$, holomorphic in $z$, such that $|v(z, s, t)| \leq 1, v(0, s, t)=0$ and

$$
f(z, s)=f(v(z, s, t), t) .
$$

Note that $|v(z, s, t)| \leq|z|$ as a consequence of Schwarz lemma. Subordination chains arise as solutions of the so called Löwner differential equation

$$
\frac{\partial}{\partial t} f(z, t)=D f(z, t)(h(z, t))
$$

where $h(z, t)$ is holomorphic in $z$, with $h(0, t)=0, D h(0, t)=\mathrm{I}$ and $\operatorname{Re}\langle\bar{z}, h(z, t)\rangle \geq 0$.

In connection to univalence and quasiconformal extension, subordination chains were originally studied in the plane by Pommerenke $[\mathbf{P}]$ and Becker [B], and later by Pfaltzgraff in higher dimensions [Pf 1], [Pf 2].

The estimates in (1.1) for starlike maps follow from the general theory by inserting $f$ as the initial element $f(z, 0)$ of the subordination chain

$$
f(z, t)=e^{t} f(z)
$$

which satisfies (1.3) with $h(z, t)=w(z)$. From this point of view it is also natural to consider what we would like to call strongly starlike maps. Let $z \in \partial B^{n}$ and let $\zeta \in B^{1}$. Then

$$
0 \leq \operatorname{Re}\langle\overline{\zeta z}, w(\zeta z)\rangle=\operatorname{Re} \sum \bar{\zeta} \bar{z}_{i} w_{i}(\zeta z)=|\zeta|^{2} \operatorname{Re} \sum \bar{z}_{i} \frac{w_{i}(\zeta z)}{\zeta}
$$

The function

$$
g(\zeta)=\sum \bar{z}_{i} \frac{w_{i}(\zeta z)}{\zeta}
$$


is holomorphic because $w(0)=0$ and has non-negative real part. Furthermore, since $D w(0)=I$ it follows that $g(0)=1$. We say that $f$ is strongly starlike if the values of $g$ lie on a fixed compact subset of the right half-plane, independent of $z$. This is equivalent to saying that

$$
g(\zeta)=\frac{1+\sigma(\zeta)}{1-\sigma(\zeta)}
$$

where $\sigma(0)=0$ and $|\sigma(\zeta)| \leq c<1, c$ an absolute constant.

For strongly starlike maps the following estimates hold:

$$
\frac{|z|}{(1+c|z|)^{2}} \leq|f(z)| \leq \frac{|z|}{(1-c|z|)^{2}}
$$

In addition, $f$ will admit a quasiconformal extension to $\mathbb{C}^{n}$ if it is already quasiconformal in $B^{n}$. This extra hypothesis guarantees a Lipschitz continuous extension to the closed ball. The extension for $|z|>1$ is given by $|z| f(z /|z|)$, which is Lipschitz continuous as well. In the plane, quasiconformality in the disc is, of course, not an issue and a quasiconformal extension exists simply as a consequense of the strong starlikeness.

In the last section we will discuss in some detail several examples of starlike and strongly starlike mappings. We shall also present a way of efficiently computing the normalized map $f$ for a given $w$.

2. Subordination chains and growth. Let $f$ be starlike, so that (1.5) holds with $|\sigma(\zeta)| \leq c \leq 1$, and let $w=(D f)^{-1}(f)$. The next lemma is due to Pfaltzgraff (Lemma 2.1, [Pf 1]). The proof, which we shall omit, is based on an application of Schwarz lemma to the function $g(\zeta)$.

LEMMA 1. The function $w$ satisfies

$$
|z|^{2} \frac{1-c|z|}{1+c|z|} \leq \operatorname{Re}\langle\bar{z}, w(z)\rangle \leq|z|^{2} \frac{1+c|z|}{1-c|z|}
$$

Let the chain $f(z, t)$ be defined by

$$
f(z, t)=e^{t} f(z) .
$$


Then

$$
\frac{\partial}{\partial t} f(z, t)=e^{t} f(z)=e^{t}(D f)(w)=D f(z, t)(h(z, t))
$$

with $h(z, t)=w(z)$. It follows now from Theorem 2.2 in [Pf 1] that $f(z, t)$ is a subordination chain. His result applies to a more general class of functions $h(z, t)$, whereas in our case one can show more or less directly that $f(z, t)$ forms a subordination chain. Let $v(z, s, t)$ be defined by

$$
v(z, s, t)=f^{-1}\left(e^{s-t} f(z)\right) .
$$

This is well-defined for $0 \leq s \leq t$ because $f$ is starlike. By construction

$$
f(z, s)=e^{s} f(z)=e^{t} f(v(z, s, t))=f(v(z, s, t), t) .
$$

We need to show that $v$ is a Schwarz function. Since $f(0)=0$ it follows that $v(0, s, t)=0$. From (2.2) we have

$$
\frac{\partial}{\partial t} v(z, s, t)=(D f)^{-1}\left(-e^{s-t} f(z)\right)=-(D f)^{-1}\left(e^{s-t} f(z)\right)
$$

where $D f$ is evaluated at $f^{-1}\left(e^{s-t} f(z)\right)$. Using starlikeness we have

$$
\frac{\partial}{\partial t} v(z, s, t)=-w\left(f^{-1}\left(e^{s-t} f(z)\right)\right)=-w(v(z, s, t)) .
$$

We compute

$$
\begin{aligned}
\frac{\partial}{\partial t}|v(z, s, t)|^{2} & =2|v(z, s, t)| \frac{\partial}{\partial t}|v(z, s, t)| \\
& =2 \operatorname{Re}\left\langle\overline{v(z, s, t)}, \frac{\partial}{\partial t} v(z, s, t)\right\rangle
\end{aligned}
$$

hence

$$
\frac{\partial}{\partial t}|v(z, s, t)|=-|v(z, s, t)|^{-1} \operatorname{Re}\langle\overline{v(z, s, t)}, w(v(z, s, t))\rangle \leq 0 .
$$

Since $v(z, s, s)=z$ we conclude that $|v(z, s, t)| \leq|z|$. 
THEOREM 1. The function $f$ satisfies

$$
\frac{|z|}{(1+c|z|)^{2}} \leq|f(z)| \leq \frac{|z|}{(1-c|z|)^{2}}
$$

Proof. Using (2.4) together with the bounds in (2.1), a simple integration yields

$$
e^{t} \frac{|v(z, s, t)|}{(1-c|v(z, s, t)|)^{2}} \leq e^{s} \frac{|z|}{(1-c|z|)^{2}}
$$

and

$$
e^{s} \frac{|z|}{(1+c|z|)^{2}} \leq e^{t} \frac{|v(z, s, t)|}{(1+c|v(z, s, t)|)^{2}}
$$

(see Lemma 2.2 in [Pf 1]). Observe that, as a consequence of the normalizations on $f,(2.2)$ implies that

$$
\lim _{t \rightarrow \infty} v(z, s, t)=0
$$

and

$$
\lim _{t \rightarrow \infty} e^{t} v(z, s, t)=e^{s} f(z) .
$$

The bounds in (2.5) follow now by taking the limit in (2.6) and (2.7).

\section{Extensions to $\overline{B^{n}}$ and $\mathbb{C}^{n}$.}

THEOREM 2. Let $f$ be a strongly starlike map of $B^{n}$ and suppose that $f$ admits a continuous extension to the closure $\overline{B^{n}}$. Then the extension is univalent in $\overline{B^{n}}$.

Proof. We follow Pfaltzgraff. Let $f(z, t)$ be the associated subordination chain with Schwarz functions $v(z, s, t), 0 \leq s \leq t$. The estimate (2.6) implies that $\overline{v\left(B^{n}, s, t\right)} \subset B^{n}$ when $s<t$. If $f$ admits a continuous extension to the closed ball, then for $s<t$, $f\left(\overline{B^{n}}, s\right) \subset f\left(B^{n}, t\right)$ as a consequence of the relation (2.3). This enables us to define a continuous extension of $v(z, s, t)$ to $\overline{B^{n}}$ via the equation

$$
v(z, s, t)=f^{-1}(f(z, s), t)
$$


For $|z|<1$,

$$
\begin{aligned}
|z-v(z, s, t)| & =\left|\int_{s}^{t} h(v(z, s, \tau), \tau) d \tau\right| \\
& \leq \int_{s}^{t}|v| \frac{1+c|v|}{1-c|v|} d \tau \leq \frac{1+c}{1-c}(t-s) .
\end{aligned}
$$

It is easy to see that this estimate holds as well for $|z| \leq 1$. We can now show that $f$ is univalent in the closed ball. Suppose that $f\left(z_{1}\right)=f\left(z_{2}\right)$ for $z_{1}, z_{2} \in \overline{B^{n}}$. Then for $t>0$

$$
f\left(z_{1}\right)=f\left(v\left(z_{1}, 0, t\right), t\right)=f\left(v\left(z_{2}, 0, t\right), t\right)=f\left(z_{2}\right),
$$

and since $f$ is univalent in $B^{n}$ we conclude that

$$
v\left(z_{1}, 0, t\right)=v\left(z_{2}, 0, t\right)
$$

By letting $t \rightarrow 0,(3.1)$ implies that $z_{1}=z_{2}$.

Unlike in the case $n=1$, when a holomorphic map in the unit disc is trivially quasiconformal, this additional assumption becomes essential when studying quasiconformal extensions in higher dimensions. Recall that a map $f: \Omega \subset \mathbb{R}^{m} \rightarrow \mathbb{R}^{m}$ is said to be quasiconformal if it is differentiable a.e., ACL (absolutely continuous on lines) for almost all lines, and

$$
\|D f\|^{m} \leq K|\operatorname{det} D f| \text { a.e. } \Omega \text {. }
$$

Here $\|D f\|$ stands for the usual norm

$$
\|D f\|=\sup \{|D f(X)|:|X|=1\}
$$

and the constant $K$ is called the quasiconformal distortion of the $\operatorname{map} f$.

THEOREM 3. Let $f$ be a quasiconformal, strongly starlike map of $B^{n}$ with $|w|$ uniformly bounded. Then $f$ admits a Lipschitz continuous extension to $\overline{B^{n}}$ and a quasiconformal extension to $\mathbb{C}^{n}$.

Proof. We first show that $\|D f\|$ is uniformly bounded in $B^{n}$. This will imply that $f$ admits a Lipschitz continuous extension to the closed ball. 
The first inequality in (2.1) together with the Cauchy-Schwarz inequality imply that

$$
|z| \frac{1-c|z|}{1+c|z|} \leq|w|
$$

Since $D f(w)=f$, it follows from (2.5) that

$$
\left|D f\left(\frac{w}{|w|}\right)\right| \leq \frac{1+c|z|}{(1-c|z|)^{3}} \leq \frac{1+c}{(1-c)^{3}} .
$$

Note that the Cauchy-Riemann equations guarantee that, with the canonical identifications, $D f(w)=f$ remains true in the real sense. Since $f$ is quasiconformal in $B^{n}$, we conclude that for some constant $K$ and all unit vectors $X$,

$$
|D f(X)| \leq K \frac{1+c}{(1-c)^{3}} .
$$

This proves that $\|D f\|$ is uniformly bounded in the ball. (Because $|w|$ is uniformly bounded, a similar argument implies that the infimum of $|D f(X)|$ over all unit vectors is bounded below by some positive constant, and therefore $f$ is actually quasi-isometric in $B^{n}$.)

Let us still denote by $f$ the extension to $\overline{B^{n}}$ and define $F: \mathbb{R}^{2 n} \rightarrow$ $\mathbb{R}^{2 n}$ by

$$
F(z)= \begin{cases}f(z), & |z| \leq 1 \\ |z| f\left(\frac{z}{|z|}\right), & |z|>1\end{cases}
$$

This definition is natural from the point of view of subordination chains. We claim that $F$ is the desired quasiconformal extension of $f$. It is clear that $F$ is continuous in $\mathbb{R}^{2 n}$. Note that

$$
F(z)=\left\{\begin{array}{ll}
f(z, 0), & |z| \leq 1 \\
f\left(\frac{z}{|z|}, \log |z|\right), & |z|>1
\end{array} .\right.
$$

Since $f\left(\overline{B^{n}}, s\right) \subset f\left(B^{n}, t\right)$ for $0 \leq s<t$ and since $f$ is univalent in the closed ball, we conclude that, in any case, $F$ is univalent in $\mathbb{R}^{2 n}$. In order to show that $F$ is quasiconformal, we follow a standard 
argument of dilation (see, e.g., [B], [Pf 2]). For $r<1$ we consider the functions $F_{r}$ defined by

$$
F_{r}(z)= \begin{cases}f(r z), & |z| \leq 1 \\ |z| f\left(r \frac{z}{|z|}\right), & |z|>1\end{cases}
$$

where $0<r<1$.

Since $f$ is Lipschitz continuous in the closed ball, it is easy to see that, as $r$ tends to $1, F_{r} \rightarrow F$ uniformly in $\overline{B^{n}}$. It follows that the convergence is uniform on compact subsets of $\mathbb{R}^{2 n}$. We are left to show that $F_{r}$ is quasiconformal in $\mathbb{R}^{2 n}$ and that the quasiconformal distortion is uniformly bounded when $r$ is close to 1 . It is clear that $F_{r}$ is differentiable away from the set $\{|z|=1\}$, therefore it is differentiable a.e.. For $|z|<1$,

$$
D F_{r}(z)=r D f(r z)
$$

hence $\left\|D F_{r}\right\|$ is uniformly bounded in $B^{n}$. When $|z|>1$ we have

$$
D F_{r}(z)=f\left(r \frac{z}{|z|}\right)^{\mathrm{T}} \operatorname{grad}|z|+r|z| D f\left(r \frac{z}{|z|}\right)\left(|z|^{-1} \mathrm{I}-|z|^{-3} z^{\mathrm{T}} z\right) .
$$

We explain the notation. Using the standard identification of $\mathbb{C}^{n}$ with $\mathbb{R}^{2 n}$, a complex vector $a$ is written in real components $\left(a_{i}\right)$. Then $a^{\mathrm{T}} b$ stands for the $(2 n) \times(2 n)$ matrix with entries $a_{i} b_{j}$. Since $|f|,|| D f||$ and $\operatorname{grad}|z|=|z|^{-1} z$ are uniformly bounded, equation (3.5) shows that so is $\left\|D F_{r}\right\|$ for $|z|>1$. From the continuity of $F_{r}$ everywhere it follows that this map is Lipschitz continuous in $R^{2 n}$, and hence ACL a.e.

Because $f$ is quasiconformal in $B^{n}$, (3.4) implies that the same holds for $F_{r}$. Furthermore, the quasiconformal distortions in $B^{n}$ are the same. For $|z|>1$ we use (3.5). Let $\zeta=r|z|^{-1} z$. Then

$$
\begin{aligned}
D F_{r}(z) & =D f(\zeta)\left\{\frac{1}{r} w(\zeta)^{\mathrm{T}} \zeta+r\left(\mathrm{I}-\frac{1}{r^{2}} \zeta^{\mathrm{T}} \zeta\right)\right\} \\
& =D f(\zeta)\left\{r \mathrm{I}+\frac{1}{r}(w(\zeta)-\zeta)^{\mathrm{T}} \zeta\right\} \\
& =r D f(\zeta)\left\{\mathrm{I}+\frac{1}{r^{2}}(w(\zeta)-\zeta)^{\mathrm{T}} \zeta\right\}
\end{aligned}
$$


Let $\mathrm{M}=\mathrm{M}(\zeta)$ be the matrix $r^{-2}(w(\zeta)-\zeta)^{\mathrm{T}} \zeta$. It is easy to see that $\|\mathrm{M}\|=r^{-2}|w(\zeta)-\zeta||\zeta|$, which is uniformly bounded by the assumption on $w$. Also, since $M$ has rank 1 ,

$$
\begin{aligned}
\operatorname{det}(\mathrm{I}+\mathrm{M}) & =1+\operatorname{tr} \mathrm{M}=1+r^{-2} \operatorname{Re}\langle\bar{\zeta}, w(\zeta)-\zeta\rangle \\
& =r^{-2} \operatorname{Re}\langle\bar{\zeta}, w(\zeta)\rangle \\
& \geq r^{-2}|\zeta|^{2} \frac{1-c|\zeta|}{1+c|\zeta|} \geq \frac{1-c}{1+c}
\end{aligned}
$$

Equation (3.6) gives

$$
\begin{aligned}
\left\|D F_{r}(z)\right\| & \leq r\|D f(\zeta)\|\|\mathrm{I}+\mathrm{M}\| \\
& \leq r\|D f(\zeta)\|(1+\|\mathrm{M}\|) \leq K_{1}\|D f(\zeta)\|
\end{aligned}
$$

for some absolute constant $K_{1}$. It also follows from (3.6) that

$$
\begin{aligned}
\left|\operatorname{det} D F_{r}(z)\right| & =r^{2 n}|\operatorname{det} D f(\zeta)||\operatorname{det}(\mathrm{I}+\mathrm{M})| \\
& \geq r^{2 n} \frac{1-c}{1+c}|\operatorname{det} D f(\zeta)| .
\end{aligned}
$$

Finally, since $f$ is quasiconformal in $B^{n},(3.7)$ and (3.8) imply

$$
\begin{aligned}
\left\|D F_{r}(z)\right\|^{2 n} & \leq K_{1}^{2 n}\|D f(\zeta)\|^{2 n} \leq K K_{1}^{2 n}|\operatorname{det} D f(\zeta)| \\
& \leq r^{-2 n} \frac{1+c}{1-c} K K_{1}^{2 n}\left|\operatorname{det} D F_{r}(z)\right|
\end{aligned}
$$

This shows that $F_{r}$ is quasiconformal for positive $r$ and that, for $r$ close to 1 , the quasiconformal distortion is uniformly bounded. The proof is now finished.

In dimension 1 , it is not necessary to assume quasiconformality in the ball or uniform boundedness of $|w|$. The first condition is automatically satisfied with $K=1$ and the second one can be established from the strong starlikeness using Schwarz lemma.

4. Some examples. This section will be devoted to presenting examples of starlike and strongly starlike mappings. We will also address the question of existence and uniqueness of the map $f$ with $(D f)^{-1}(f)=w$, where $w$ is a given normalized function satisfying $\operatorname{Re}\langle\bar{z}, w\rangle \geq 0$. 
EXAMPLE 1. Let $f\left(z_{1}, z_{2}\right)=\left(z_{1}, z_{2}-z_{1} z_{2}\right)$. Then

$$
D f=\left(\begin{array}{cc}
1 & 0 \\
-z_{2} & 1-z_{1}
\end{array}\right)
$$

and

$$
(D f)^{-1}(f)=w=\left(z_{1}, \frac{z_{2}}{1-z_{1}}\right) .
$$

Recall the function $g$ in (1.4) given by $g(\zeta)=\sum \bar{z}_{i} \frac{w_{i}(\zeta z)}{\zeta}$, where $z \in \partial B^{n}$ and $\zeta \in B^{1}$. Here

$$
g(\zeta)=\overline{z_{1}} \frac{\zeta z_{1}}{\zeta}+\overline{z_{2}} \frac{\zeta z_{2}}{\zeta\left(1-\zeta z_{1}\right)}=\frac{1-\left|z_{1}\right|^{2} z_{1} \zeta}{1-z_{1} \zeta}
$$

and it can be written in the form $\frac{1+\sigma}{1-\sigma}$ where

$$
\sigma(\zeta)=\frac{g(\zeta)-1}{g(\zeta)+1}=\frac{\left(1-\left|z_{1}\right|^{2}\right) z_{1} \zeta}{2-\left(1+\left|z_{1}\right|^{2}\right) z_{1} \zeta}
$$

Hence

$$
|\sigma(\zeta)| \leq \frac{\left|z_{1}\right|\left(1-\left|z_{1}\right|^{2}\right)}{2-\left|z_{1}\right|\left(1+\left|z_{1}\right|^{2}\right)}=\frac{\left|z_{1}\right|\left(1+\left|z_{1}\right|\right)}{2+\left|z_{1}\right|\left(1+\left|z_{1}\right|\right)} \leq \frac{1}{2}
$$

It follows that $f$ is strongly starlike. The map $f$ is Lipschitz continuous but not quasiconformal in $B^{2}$ : the eigenvalues of $D f$ are 1 and $1-z_{1}$ and their ratio is not uniformly bounded. Observe also that $\|w\|_{\infty}=\infty$.

EXAMPLE 2. Let $f\left(z_{1}, z_{2}\right)=\left(z_{1}, z_{2}\left(1-z_{1}^{3}\right)^{-1 / 3}\right)$. Then

$$
D f=\left(\begin{array}{cc}
1 & 0 \\
\frac{z_{1}^{2} z_{2}}{\left(1-z_{1}^{3}\right)^{4 / 3}} & \frac{1}{\left(1-z_{1}^{3}\right)^{1 / 3}}
\end{array}\right)
$$

and

$$
(D f)^{-1}(f)=w=\left(z_{1}, \frac{z_{2}\left(1-2 z_{1}^{3}\right)}{1-z_{1}^{3}}\right) .
$$

In this case $g$ is given by

$$
g(\zeta)=\left|z_{1}\right|^{2}+\frac{\left|z_{2}\right|^{2}\left(1-2 z_{1}^{3} \zeta^{3}\right)}{1-z_{1}^{3} \zeta^{3}}
$$


and the corresponding function $\sigma$ is easily computed to be

$$
\sigma(\zeta)=-\frac{\left(1-\left|z_{1}\right|^{2}\right) z_{1}^{3} \zeta^{3}}{2-\left(1+\left|z_{1}\right|^{2}\right) z_{1}^{3} \zeta^{3}}
$$

Hence

$$
|\sigma(\zeta)| \leq \frac{\left|z_{1}\right|^{3}\left(1-\left|z_{1}\right|^{2}\right)}{2-\left|z_{1}\right|^{3}\left(1+\left|z_{1}\right|^{2}\right)}=\frac{\left|z_{1}\right|^{3}\left(1+\left|z_{1}\right|\right)}{2+\left|z_{1}\right|\left(1+\left|z_{1}\right|\right)\left(2+\left|z_{1}\right|^{2}\right)} \leq \frac{1}{4} .
$$

This shows that $f$ is strongly starlike. This time, $f$ is not Lipschitz continuous and, as before, the map fails to be quasiconformal. Again, $\|w\|_{\infty}=\infty$.

EXAMPLE 3. A vast class of strongly starlike mappings with uniformly bounded $w$ can be obtained by prescribing $w$ in the form

$$
w(z)=\left(\mathrm{I}+\mathrm{E}_{z}\right)^{-1}\left(\mathrm{I}-\mathrm{E}_{z}\right)(z),
$$

where $\mathrm{E}_{z}$ is an $n \times n$ matrix depending holomorphically in $z$ with $\mathrm{E}_{0}=0$ and $\left\|\mathrm{E}_{z}\right\| \leq c<1$. (For convenience we shall simply write $\mathrm{E}$ for $\mathrm{E}_{z}$.) Indeed,

$$
w+z=(\mathrm{I}+\mathrm{E})^{-1}\{(\mathrm{I}-\mathrm{E})(z)+(\mathrm{I}+\mathrm{E})(z)\}=2(\mathrm{I}+\mathrm{E})^{-1}(z)
$$

and similarly

$$
w-z=-2 \mathrm{E}(\mathrm{I}+\mathrm{E})^{-1}(z)=-\mathrm{E}(w+z),
$$

hence

$$
|w-z|^{2} \leq\|\mathrm{E}\|^{2}|w+z|^{2}
$$

The general form of Schwarz lemma implies that $\|\mathrm{E}\| \leq c|z|$ and so

$$
|w-z|^{2} \leq c^{2}|z|^{2}|w+z|^{2} .
$$

From this we conclude that

$$
|z|^{2} \frac{1-c|z|}{1+c|z|} \leq \operatorname{Re}\langle\bar{z}, w\rangle \leq|z|^{2} \frac{1+c|z|}{1-c|z|}
$$

where the right hand inequality is a consequence of the definition of $w$ and the Cauchy-Schwarz inequality. This shows that a mapping $f$ with $(D f)^{-1}(f)=w$ is strongly starlike. But can $w$ with $\operatorname{Re}\langle\bar{z}, w\rangle \geq$ 
0 be prescribed arbitrarily? In dimension 1 the solution $f$ can be found explicitly by integration. In higher dimension we consider the flows

$$
\frac{d z}{d t}=w(z)
$$

and

$$
\frac{d F}{d t}=F
$$

The condition of starlikeness, $(D f)(w)=f$, translates to the fact that $f$ takes integral curves of (4.2) to those of (4.3). We will call such a map a conjugation of flows. Both vector fields in question, $w$ and the identity, have an isolated critical point at 0 and moreover, the latter is simply the linear part of $w$ at the origin. Conjugating a given flow to its linear part at a critical point is a classical problem in the theory of stability of ordinary differential equations, and the existence of a (local) conjugating map depends on the nature of the eigenvalues of the aforementioned linear part. Depending also on the degree of smoothness of the vector field, it is a problem that can be posed in the $\mathbb{C}^{k}, \mathbb{C}^{\infty}$ or holomorphic category $([\mathbf{H}])$.

In his thesis, Poincaré answered the question for holomorphic $w$ and his result applies in our particular and simplest case when all eigenvalues are equal to 1 ([A, p.181]). The solution $f$ is a biholomorphism in a neighborhood of the origin and can be extended to the ball since $w$ is holomorphic there and has no other critical points.

Poincaré's proof consists of first showing that there exists a formal power series solution and then proving convergence of the resulting series. When $w$ is given explicitly, there is an often more effective way of computing the map $f$. We will obtain $f$ as a limit of conjugating maps, and since for the moment we are interested in constructing examples only, certain facts will be claimed here without proofs. Because the vector field $w$ is transverse to centered spheres, for $\epsilon \leq|z|<1$ we can define uniquely a continuous map $f_{\epsilon}$ by the conditions:

(1) $f_{\epsilon}(z)=z$ for $|z|=\epsilon$

(2) $f_{\epsilon}$ conjugates the flows for $|z|>\epsilon$. 
These maps are quite easy to deal with as the flows now have no critical points. A simple integration together with Lemma 1 yields the estimates

$$
\frac{(1+\epsilon c)^{2}|z|}{(1+c|z|)^{2}} \leq\left|f_{\epsilon}(z)\right| \leq \frac{(1-\epsilon c)^{2}|z|}{(1-c|z|)^{2}}
$$

Using the fact that $\operatorname{Re}\langle\bar{z}, w\rangle \geq 0$ and that $D w(0)=\mathrm{I}$ it can be shown that as $\epsilon \rightarrow 0,\left\{f_{\epsilon}\right\}$ converges uniformly on compact subsets of the punctured ball. In addition, the limiting map $f$ satisfies

$$
|f(z)-z|=o(|z|) \text {. }
$$

Therefore, $f$ can be extended as a differentiable map at 0 , with $D f(0)=\mathrm{I}$. By construction, $f$ is a conjugation and we conclude that it must be the desired biholomorphisms. (If $F_{1}, F_{2}$ are two conjugations with $D F_{1}(0)=D F_{2}(0)$ then $G=F_{1} \circ F_{2}^{-1}$ conjugates the flow along rays from the origin to itself. Since $D G(0)=I$ it is easy to show that $G$ must be the identity, thus $F_{1}=F_{2}$.) With this, (4.4) gives back the growth for starlike and strongly starlike mappings.

EXAMPLE 4. Let $w\left(z_{1}, z_{2}\right)=\left(z_{1}\left(1+z_{2}\right), z_{2}\left(1+z_{1}\right)\right)$. Then $w$ is normalized and

$$
\operatorname{Re}\langle\bar{z}, w\rangle=\left|z_{1}\right|^{2} \operatorname{Re}\left(1+z_{2}\right)+\left|z_{2}\right|^{2} \operatorname{Re}\left(1+z_{1}\right) \geq 0 .
$$

The equations for the $w$-flow are

$$
\frac{d z_{1}}{d t}=z_{1}\left(1+z_{2}\right) \quad \text { and } \quad \frac{d z_{2}}{d t}=z_{2}\left(1+z_{1}\right)
$$

and can be solved by first eliminating the parameter. If the initial conditions are $z_{1}(0)=\epsilon a$ and $z_{2}(0)=\epsilon b,|a|^{2}+|b|^{2}=1$, then one finds

$$
z_{1}(t)=\frac{\epsilon a(a-b) e^{t}}{a-b e^{\epsilon(b-a)} e^{\epsilon(a-b) e^{t}}}
$$

and

$$
z_{2}(t)=\frac{\epsilon b(b-a) e^{t}}{b-a e^{\epsilon(a-b)} e^{\epsilon(b-a) e^{t}}}
$$


The map $f_{\epsilon}$ is defined so that

$$
f_{\epsilon}\left(z_{1}(t), z_{2}(t)\right)=\left(\epsilon a e^{t}, \epsilon b e^{t}\right) .
$$

From this we have to once again eliminate the parameter $t$ and then let $\epsilon \rightarrow 0$. With $f_{\epsilon}=\left(f_{1}, f_{2}\right)$ (we have omitted the $\epsilon$-dependence), equations (4.5), (4.6) and (4.7) give

$$
z_{1}=a \frac{f_{1}-f_{2}}{a-b e^{\epsilon(b-a)} e^{f_{1}-f_{2}}}=\frac{f_{1}\left(f_{1}-f_{2}\right)}{f_{1}-f_{2} e^{f_{1}-f_{2}} e^{\epsilon(b-a)}}
$$

and

$$
z_{2}=\frac{f_{2}\left(f_{2}-f_{1}\right)}{f_{2}-f_{1} e^{f_{2}-f_{1}} e^{\epsilon(a-b)}} .
$$

We let $\epsilon \rightarrow 0$ to obtain

$$
\begin{aligned}
& z_{1}=\frac{f_{1}\left(f_{1}-f_{2}\right)}{f_{1}-f_{2} e^{f_{1}-f_{2}}} \\
& z_{2}=\frac{f_{2}\left(f_{2}-f_{1}\right)}{f_{2}-f_{1} e^{f_{2}-f_{1}}} .
\end{aligned}
$$

To invert this system first observe that

$$
\frac{z_{2}}{z_{1}}=\frac{f_{2}}{f_{1}} e^{f_{1}-f_{2}}
$$

which inserted in (4.8) gives

$$
f_{1}-f_{2}=z_{1}-z_{2} .
$$

Equation (4.10) and (4.11) finally yield the mapping $f=\left(f_{1}, f_{2}\right)$ :

$$
\begin{aligned}
& f_{1}=\frac{z_{1}\left(z_{1}-z_{2}\right) e^{z_{1}}}{z_{1} e^{z_{1}}-z_{2} e^{z_{2}}} \\
& f_{2}=\frac{z_{2}\left(z_{2}-z_{1}\right) e^{z_{2}}}{z_{2} e^{z_{2}}-z_{1} e^{z_{1}}} .
\end{aligned}
$$

It can be checked directly that indeed $D f(w)=f$. The reader interested in verifying this may want to use (4.11) and (4.10) (after 
taking logarithms) to obtain useful relations between the partial derivatives of $f_{1}$ and $f_{2}$.

Finally we would like to mention a related problem we might address in a future paper. The first two examples in this section exhibited strongly starlike mappings that were not quasiconformal. As pointed out at the time, in both cases $\|w\|_{\infty}=\infty$. So far, we have been unable to produce an example of a strongly starlike map with finite $\|w\|_{\infty}$ which is not quasiconformal. A proof that all strongly starlike mappings with $\|w\|_{\infty}<\infty$ are quasiconformal has also eluded us, even in the case when $w$ is given as in (4.1). There are some partial results, nevertheless. The map $f$ will be quasiconformal provided the mappings $f_{\epsilon}$ are uniformly quasiconformal, at least for small $\epsilon$. This is the case when, for instance, $w$ is given as in (4.1) and the entries of $\mathrm{E}$ have uniformly bounded derivatives.

Acknowledgments. J. Pfaltzgraff has kindly informed us of his talk at the January 1990 AMS meeting in Louisville, where he mentioned the fact that the growth theorem for starlike mappings obtained by Barnard, FitzGerald and Gong, could be derived from his work on subordination chains in several variables, much like it was presented here.

I would also like to thank Ian Graham, who brought to my attention the result of Barnard, FitzGerald and Gong during my visit of Toronto in February of 92. Later conversations with him and his suggestions on some of the references were most helpful.

\section{REFERENCES}

[A] V. Arnold, Geometrical Methods in the Theory of Ordinary Differential Equations, Springer-Verlag, New York, 1987.

BFG 1] R. Barnard, C. FitzGerald and S. Gong, The growth and 1/4-theorems for starlike mappings in $\mathbb{C}^{n}$, Pacific J. Math., 150 (1991), 13-22.

BFG 2] _ $\quad A$ distortion theorem for biholomorphic mappings in $\mathbb{C}^{2}$, to appear in Trans. Amer. Math. Soc.

[B] J. Becker, Löwnersche Differentialgleichungen and quasikonform fortsetzbare Funktionen, J. Reine Angew. Math., 255 (1972), 23-43.

[D] P. Duren, Univalent Functions, Springer-Verlag, New York, 1983.

[DR] P. Duren and W. Rudin, Distortion in several variables, Complex Variables Theory Appl., 5 (1986), 323-326.

[G 1] I. Graham, Distortion theorems for holomorphic maps between convex 
domains in $\mathbb{C}^{n}$, Complex Variables Theory Appl., 15 (1990), 37-42.

[G 2] Sharp constants for the Koebe theorem and for estimates of intrinsic metrics on convex domains, Proc. Symp. Pure Math. 52, Several Complex variables and Complex Geometry, part 2, pp. 233-238, Amer. Math. Soc., Providence, 1991.

[H] P. Hartman, Ordinary Differential Equations, Birkhäuser, Boston, 1982.

[LV] O. Lehto and K. Virtanen, Quasiconformal Mappings in the Plane, Springer-Verlag, Berlin, 1973.

[L] K. Löwner, Untersuchungen über schlichte konforme Abbildungen des Einheitskreises, Math. Ann., 89 (1923), 103-121.

[Pf 1] J. Pfaltzgraff, Subordination chains and univalence of holomorphic mappings in $\mathbb{C}^{n}$, Math. Ann., 210 (1974), 55-68.

[Pf 2] - Subordination chains and quasiconformal extension of holomorphic maps in $\mathbb{C}^{n}$, Ann. Acad. Scie. Fenn. Ser. A I Math., 1 (1975), 13-25.

[Pf-S] J. Pfaltzgraff and T. Suffridge, Close-to-starlike holomorphic functions of several variables, Pacific J. Math., 57 (1975), 271-279.

[P] C. Pommerenke, Über die Subordination analytischer Funktionen, J. Reine Angew. Math., 218 (1965), 159-173.

[R] M. Robertson, Applications of the subordination principle to univalent functions, Pacific J. Math., 11 (1961), 315-324.

[S 1] T. Suffridge, The principle of subordination applied to functions of several complex variables, Pacific J. Math., 33 (1970), 241-248.

[S 2] , Starlike and convex maps in Banach spaces, Pacific J. Math., 48 (1973), 575-589.

[V] J. Väisälä, Lectures on n-Dimensional Quasiconformal Mappings, Lecture Notes in Math. 229, Springer-Verlag, New York, 1971.

Received August 26, 1992.

P. Universidad Católica de Chile

Casilla 306, Santiago 22

CHILE

E-mail address: mchuaqui@mat.puc.cl 


\section{PACIFIC JOURNAL OF MATHEMATICS}

Founded by E. F. Beckenbach (1906-1982) and F. Wolf (1904-1989)

\section{EDITORS}

Sun-Yung Alice Chang (Managing Editor)

University of California

Los Angeles, CA 90095-1555

pacific@math.ucla.edu

F. Michael Christ

University of California

Los Angeles, CA 90095-1555

christ@math.ucla.edu

Thomas Enright

University of California

San Diego, La Jolla, CA 92093

tenright@ucsd.edu

Nicholas Ercolani

University of Arizona

Tucson, AZ 85721

ercolani@math.arizona.edu
Robert Finn

Stanford University

Stanford, CA 94305

finn@gauss.stanford.edu

Vaughan F. R. Jones

University of California

Berkeley, CA 94720

vfr@math.berkeley.edu

Steven Kerckhoff

Stanford University

Stanford, CA 94305

spk@gauss.stanford.edu

\author{
Martin Scharlemann \\ University of California \\ Santa Barbara, CA 93106 \\ mgscharl@math.ucsb.edu \\ Gang Tian \\ Courant Institute \\ New York University \\ New York, NY 10012-1100 \\ tiang@taotao.cims.nyu.edu
V. S. Varadarajan
University of California
Los Angeles, CA 90095-1555
vsv@math.ucla.edu

\section{SUPPORTING INSTITUTIONS}

\section{CALIFORNIA INSTITUTE OF TECHNOLOGY NEW MEXICO STATE UNIVERSITY OREGON STATE UNIVERSITY \\ STANFORD UNIVERSITY \\ UNIVERSITY OF ARIZONA \\ UNIVERSITY OF BRITISH COLUMBIA \\ UNIVERSITY OF CALIFORNIA UNIVERSITY OF HAWAII}

\author{
UNIVERSITY OF MONTANA \\ UNIVERSITY OF NEVADA, RENO \\ UNIVERSITY OF OREGON \\ UNIVERSITY OF SOUTHERN CALIFORNIA \\ UNIVERSITY OF UTAH \\ UNIVERSITY OF WASHINGTON \\ WASHINGTON STATE UNIVERSITY
}

The supporting Institutions listed above contribute to the cost of publication of this Journal, but they are not owners or publishers and have no responsibility for its contents or policies.

Manuscripts must be prepared in accordance with the instructions provided on the inside back cover.

The Pacific Journal of Mathematics (ISSN 0030-8730) is published monthly except for July and August. Regular subscription rate: $\$ 215.00$ a year (10 issues). Special rate: $\$ 108.00$ a year to individual members of supporting institutions.

Subscriptions, orders for back issues published within the last three years, and changes of subscribers address should be sent to Pacific Journal of Mathematics, P.O. Box 4163, Berkeley, CA 94704-0163, U.S.A. Prior back issues are obtainable from Kraus Periodicals Co., Route 100, Millwood, NY 10546.

The Pacific Journal of Mathematics at the University of California, c/o Department of Mathematics, 981 Evans Hall, Berkeley, CA 94720 (ISSN 0030-8730) is published monthly except for July and August. Second-class postage paid at Berkeley, CA 94704, and additional mailing offices. POSTMASTER: send address changes to Pacific Journal of Mathematics, P.O. Box 6143, Berkeley, CA 94704-0163.

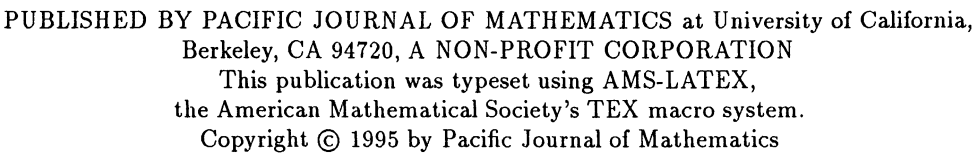




\section{PACIFIC JOURNAL OF MATHEMATICS}

Volume $168 \quad$ No. $1 \quad$ March 1995

Compact contractible $n$-manifolds have arc spines $(n \geq 5)$

FREDRIC DAVIS ANCEL and CRAIG R. GUILBAULT

On uniform homeomorphisms of the unit spheres of certain Banach lattices

\section{FOUAD CHAATIT}

Applications of subordination chains to starlike mappings in $\mathbb{C}^{n}$

MARTIN CHUAQUi

The $L^{p}$ theory of standard homomorphisms

FEREIDOUn GHAHRAMANI and SANDY GRABINER

Metrics for singular analytic spaces

Caroline Galt Grant and Pierre Milman

Maps on infra-nilmanifolds

KYUNG BAI LEE

Hardy spaces and oscillatory singular integrals. II

YIBIAO PAN

Continuity of convex hull boundaries

Linda Jo Goldway KeEn and Caroline SERIES 